\title{
Generalized Common Fixed Point Result in Banach Space
}

\author{
Kanhaiya Jha \\ School of Science, Kathmandu University, Nepal \\ Correspondence to: Kanhaiya Jha, email: jhakn@ku.edu.np
}

\begin{abstract}
The aim of this paper is to establish a common fixed point theorem for weakly compatible pair of self mappings in Banach space which generalizes and improves various well known comparable results.
\end{abstract}

Keywords: Fixed point, Banach space, compatible mappings.

\section{Introduction}

The study of common fixed points of mappings satisfying certain contractive type conditions has been a very active field of research. In 1982, Sessa [12] defined weak commutativity and proved a common fixed point theorem for weakly commuting mappings. Also, Jungck 44 introduced more generalized commutativity, so-called compatibility, which is more general than that of weak commutativity. Further, in 1998, Jungck and Rhoades [5] introduced the notion of weakly compatible and showed that compatible maps are weakly compatible but the converse need not be true.

Some theorems on unique fixed points for expansive mappings are proved by Popa [8]. Popa 9 further extended results 8 for compatible mappings. In 1999, Popa [10] proved some common fixed point theorems for compatible mappings satisfying an implicit relation. For more common fixed point results in metric space using implicit relation, one can refer Aliouche et al. 1], Imdad et al. [3, Kumar and Jha [6, Pathak et al. [7] and Popa [11.

The main purpose of this paper is to prove a common fixed point theorem for weakly compatible mappings in Banach Space satisfying an implicit relation without employing continuity condition. Our result generalizes and improves various other similar results of fixed points. We also give an example to illustrate our result.

Definition 1.1 (Weak Compatibility of self-mappings)

Two self mappings $\mathrm{A}$ and $\mathrm{S}$ of a metric space (X, d) are said to be weakly compatible if they commute at coincident points. That is, $\mathrm{Ax}=\mathrm{Sx}$ implies that $\mathrm{ASx}=\mathrm{SAx}$ for all $\mathrm{x}$ in $\mathrm{X}$. Lemma 1.14] Let $\mathrm{S}$ and $\mathrm{T}$ be weakly compatible self mappings on a metric space (X,d). If $\mathrm{St}=\mathrm{Tt}$ for some $t \in X$ then we have

$$
S T t=T S t .
$$

We start with the following implicit relations:

Let $\Phi$ be the set of all real continuous functions $\phi\left(t_{1}, t_{2}, \cdots, t_{6}\right)=\mathbb{R}_{+}^{6} \rightarrow \mathbb{R}$ satisfying the following conditions:

$\phi_{1}: \phi$ is non increasing in variable $t_{6}$.

$\phi_{2}$ : there exists $h \in(0,1)$ such that for every $u, v \geq 0$ with

$\phi_{a}: \phi(u, v, v, u,(u+v) / 2,0) \leq 0$

or

$\phi_{b}: \phi(u, v, u, v,(u+v) / 2, u+v) \geq 0$, we have $u \leq h v$.

$\phi_{3}: \phi(u, u, 0,0,0, u)>0$ for all $u>0$.

\section{Example 1.1}

$\phi\left(t_{1}, t_{2}, \cdots, t_{6}\right)=t_{1}-k \max \left\{t_{2}, t_{3}, t_{4}, t_{5},(1 / 2) t_{6}\right\}$ where $k \in(0,1)$.

$\phi_{1}$ : obvious.

$\phi_{2}$ : Let $u>0, \phi(u, v, v, u,(u+v) / 2,0)=u-k \max \{v, v, u,(u+v) / 2,0\} \leq 0$. If $u \geq v$, then $u \leq k u<u, a$ contadiction.

Thus, we have $u<v$ and $u \leq k u=h v$ where $h=k \in(0,1)$

Similarly, if $u>0$ then $\phi(u, v, v, u,(u+v) / 2,0) \leq 0$ which implies that $u \leq h v$. Also, if $u=0$, then $u \leq h v$. 
$\phi_{3}: \phi(u, u, 0,0,0, u)=(1-k) u>0$ for all $u>0$.

The following examples can be used the support above lemma.

\section{Example 1.2}

$\phi\left(t_{1}, t_{2}, \cdots, t_{6}\right)=t_{1}^{2}-\alpha\left\{t_{2}^{2}-t_{6}\left((1 / 2)\left(t_{3}+t_{4}\right)-t_{5}\right)\right\}$ where $\alpha \in(0,1)$ where $k \in(0,1)$.

$\phi_{1}$ : obvious.

$\phi_{2}$ : Let $u>0, \phi(u, v, v, u,(u+v) / 2,0)=u^{2}-a v^{2} \leq 0$ which implies that $u \leq v \sqrt{a}=h v$ where $h=\sqrt{a}<1$. Similarly, if $u>0$ then $\phi(u, v, v, u,(u+v) / 2,0) \leq 0$ which implies that $u \leq h v$. Also, if $u=0$, then $u \leq h v$. $\phi_{3}: \phi(u, u, 0,0,0, u)=u^{2}(1-a)>0$ for all $u>0$.

\section{Example 1.3}

$$
\phi\left(t_{1}, t_{2}, \cdots, t_{6}\right)=t_{1}^{2}-c_{1} \max \left\{t_{2}^{2}, t_{3}^{2}, t_{4}^{2}\right\}
$$

$-c_{2} \max \left\{t_{3} t_{5},(1 / 2)\left(t_{4} t_{6}\right)\right\}$ where $c_{1}>0, c_{2}>0, c_{1}+c_{2}<1$.

$\phi_{1}$ : obvious.

$\phi_{2}$ : Let $u>0, \phi(u, v, v, u,(u+v) / 2,0)=u^{2}-c_{1} \max \left\{v^{2}, v^{2}, u^{2}\right\}$

$-c_{2} \max \{v(1 / 2)(u+v), 0\} \leq 0$.

If $u \geq v$ then $u^{2}\left(1-c_{1}-c_{2}\right) \leq 0$ which implies that $c_{1}+c_{2} \geq 1$ a contradiction. Thus we have $u<v$ and $u \leq \bar{v} \sqrt{\left(c_{1}+c_{2}\right)}=h v$ where $h=\sqrt{\left(c_{1}+c_{2}\right)}<1$.

Similarly, if $u>0$ then $\phi(u, v, v, u,(u+v) / 2,0)$ implies that $u \leq h v$.

$\phi_{3}: \phi(u, u, 0,0,0, u)=u^{2}\left(1-c_{1}\right)>0$ for all $u>0$.

\section{Main results}

\section{Theorem 2.1}

Let $(X,\|\|$.$) be a Banach space and A, B, S, T: X \rightarrow X$ be mappings satisfying the conditions:

(i) $A X \subset T X$ and $B X \subset S X$, and

(ii) $\phi(\|A x-B y\|,\|S x-T y\|,\|A x-S x\|,\|B y-T y\|,(\|A x-S x\|+\|B y-T y\|) / 2$,

$\|A x-T y\|) \leq 0$, for all $\mathrm{x}, \mathrm{y}$ in $\mathrm{X}$ where $\phi \in \Phi$.

If one of SX, TX, AX or BX is a complete subspace of $\mathrm{X}$ and $\{\mathrm{A}, \mathrm{S}\}$ and $\{\mathrm{B}, \mathrm{T}\}$ are weakly compatible pairs, then $\mathrm{A}, \mathrm{B}, \mathrm{S}$ and $\mathrm{T}$ have a unique common fixed point.

Proof Since $A X \subset T X$, using condition (i) for an arbitrary point $x_{0}$ in $\mathrm{X}$ there exists a point $x_{1}$ in $\mathrm{X}$ such that $A x_{0}=T x_{1}$. Also, since $B X \subset S X$, for this point $x_{1}$ in $\mathrm{X}$ we can choose a point $x_{2}$ in $\mathrm{X}$ such that $B x_{1}=S x_{2}$, and so on. Inductively, we can define a sequences $\left\{x_{n}\right\}$ and $\left\{y_{n}\right\}$ in $\mathrm{X}$ such that $y_{2 n}=T x_{2 n+1}=A x_{2 n}$ and $y_{2 n+1}=S x_{2 n+2}=B x_{2 n+1}$ for every $n=0,1,2, \ldots$

Applying relation (ii), we have

$\phi\left(\left\|A x_{2 n}-B x_{2 n+1}\right\|,\left\|S x_{2 n}-T x_{2 n+1}\right\|,\left\|A x_{2 n}-S x_{2 n}\right\|,\left\|B x_{2 n+1}-T x_{2 n+1}\right\|\right.$,

$\left.\left(\left\|A x_{2 n}-S x_{2 n}\right\|+\left\|B x_{2 n+1}-T x_{2 n+1}\right\|\right) / 2,\left\|A x_{2 n}-T x_{2 n+1}\right\|\right) \leq 0$,

this implies

$\phi\left(\left\|A x_{2 n}-B x_{2 n+1}\right\|,\left\|A x_{2 n}-B x_{2 n-1}\right\|,\left\|A x_{2 n}-B x_{2 n-1}\right\|,\left\|A x_{2 n}-B x_{2 n+1}\right\|\right.$,

$\left.\left(\left\|A x_{2 n}-B x_{2 n-1}\right\|+\left\|A x_{2 n}-B x_{2 n+1}\right\|\right) / 2,0\right) \leq 0$.

By $\left(\phi_{a}\right)$, we have $\left\|A x_{2 n}-B x_{2 n+1}\right\| \leq h\left\|A x_{2 n}-B x_{2 n-1}\right\|$.

Similarly, applying $\left(\phi_{1}\right)$ and $\left(\phi_{b}\right)$, we get $\left\|A x_{2 n}-B x_{2 n-1}\right\| \leq h\left\|A x_{2 n-2}-B x_{2 n-1}\right\|$ and so for $n=0,1,2,3, \ldots$ we have $\left\|A x_{2 n}-B x_{2 n-1}\right\| \leq h^{2 n}\left\|A x_{0}-B x_{1}\right\|$.

It follows that $y_{n}$ is a Cauchy sequence in $\mathrm{X}$.

Now, suppose SX is complete. The sequence $\left\{y_{2 n+1}\right\}$ is contained in SX and has a limit, say u, in SX. Let $v \in S^{-1} u$. Then, we get $\mathrm{Sv}=u$. Also, the subsequence $\left\{y_{2 n}\right\}$ also converges to $\mathrm{u}$. Now, we claim that

$\mathrm{Av}=\mathrm{u}$. If $A v \neq u$, then using relation (ii), we get

$\phi\left(\left\|A v-B x_{2 n+1}\right\|,\left\|S v-T x_{2 n+1}\right\|,\|A v-S v\|,\left\|B x_{2 n+1}-T x_{2 n+1}\right\|\right.$,

$\left.\left(\|A v-S v\|+\left\|B x_{2 n+1}-T x_{2 n+1}\right\|\right) / 2,\left\|A v-T x_{2 n+1}\right\|\right) \leq 0$.

As $n \rightarrow \infty$, by the continuity of $\phi$, we get

$\phi(\|A v-u\|, 0,\|A v-u\|, 0,(\|A v-u\|+0) / 2,\|A v-u\|) \leq 0$

which implies by $\left(\phi_{b}\right)$ that $\mathrm{Av}=\mathrm{u}$. This means that $\mathrm{u}$ is in the range of $\mathrm{A}$ and since $A X \subset T X$, there exists $\mathrm{w}$ in $\mathrm{X}$ such that $\mathrm{Tw}=\mathrm{u}$.

Now, we show that $\mathrm{Bw}=\mathrm{u}$. If $B w \neq u$, then by using (ii), we have 
$\phi(\|A v-u\|,\|S v-T w\|,\|A v-S v\|,\|B w-T w\|,(\|A v-S v\|+\|B w-w\|) / 2$,

$\|A v-T w\|) \leq 0$.

As $n \rightarrow \infty$, by the continuity of $\phi$, we get

$\phi(0,0,\|u-B w\|, 0,(\|u-B w\|+0) / 2,0) \leq 0)$

which implies by $\left(\phi_{b}\right)$ that $\mathrm{Bw}=\mathrm{u}$. Thus, we have $\mathrm{Av}=\mathrm{Sv}=\mathrm{u}=\mathrm{Tw}=\mathrm{Bw}$. Since $\{\mathrm{A}, \mathrm{S}\}$ and $\{\mathrm{B}, \mathrm{T}\}$ are weakly compatible at $\mathrm{v}$ and $\mathrm{w}$ respectively, so we get

$\mathrm{Au}=\mathrm{ASv}=\mathrm{SAv}=\mathrm{Su}$ and $\mathrm{Bu}=\mathrm{BTw}=\mathrm{TBw}=\mathrm{Tu}$.

Thus, from relation (ii), we have

$\phi(\|A u-B u\|,\|S u-T u\|,\|A u-S u\|,\|B u-T u\|,(\|A u-S u\|+\|B u-T u\|) / 2$,

$\|A u-T u\|) \leq 0$.

This implies $\bar{\phi}(\|u-T u\|,\|u-T u\|, 0,0,0,\|u-T u\|) \leq 0$,

which contradicts to $\left(\phi_{3}\right)$ if $\|u-T u\| \neq 0$. Thus, we have $\mathrm{Tu}=\mathrm{u}$.

Similarly, we can prove that $\mathrm{Au}=\mathrm{u}$. Hence, we get $\mathrm{u}=\mathrm{Au}=\mathrm{Su}=\mathrm{Tu}=\mathrm{Bu}$.

We have therefore proved that $\mathrm{u}$ is a common fixed point of $\mathrm{A}, \mathrm{B}, \mathrm{S}$ and $\mathrm{T}$. Again, the uniqueness of the common fixed point follows easily using condition (ii).

This completes the proof of the theorem.

Now, we give the following example to support above result.

\section{Example 2.1}

Let $\mathrm{X}=[2,20]$ with the usual norm. Define $\mathrm{A}, \mathrm{B}, \mathrm{S}$ and $\mathrm{T}$ by

$A=B: X \rightarrow X$ by $B x=x$ if $x=2$ or $>5, B x=6$ if $2<x \leq 5$;

and $S=T: X \rightarrow X$ by $T x=x$ if $x=2, T x=12$ if $2<x \leq 5, T x=x-3$ if $x>5$.

Then, the pairs $(\mathrm{A}, \mathrm{S})$ and $(\mathrm{B}, \mathrm{T})$ are weakly compatible mappings. Also, the mappings satisfy all the conditions of above theorem and they have a unique common fixed point $\mathrm{x}=2$.

\section{Conclusion}

As the fixed point result has been established with minimal contractive condition, so it improves and generalizes the results of Popa [7, 9, 10] and Sharma and Deshpandey [13]. It also extends the result of Chugh and Kumar [2] and other similar results for fixed point in Banach space.

\section{References}

[1] Aliouche AK, Merghadi F and Fisher B (2008) Related fixed point theorem via implicit relations of integral type. Math. Sci., 2(1), 105 - 121.

[2] Chugh R and Kumar S (2001) Common fixed points for weakly compatible mappings. Proc. Indian Acad. Sci. (Math. Sci.), III(2), $241-247$.

[3] Imdad M, Kumar S and Khan MS (2002) Remarks on some fixed point theorems satisfying implicit relations. Radovi Matematiki, 11, 135 - 143.

[4] Jungck G (1986) Compatible mappings and common fixed points. Int. J. Math. Math. Sci., 9, 771 779 .

[5] Jungck G and Rhoades BE (1998) Fixed point for set valued functions without continuity, Indian J. Pure Appl. Math., 29(3), 227-238.

[6] Kumar S and Jha K (2007) Remarks on fixed points under implicit relations. The Nepali Math. Sci. Report, 27(1-2), $61-68$.

[7] Pathak HK, Lopez RR and Verma RK (2007) A common fixed point using implicit relation and property (E.A) in metric space. Filomat, 21(2), 211 - 234.

[8] Popa V (1990) Theorems of unique fixed point for expansion mappings. Demonstratio Math., 23, 213 $-218$. 
[9] Popa V (1993) Common fixed point of compatible mappings. Demonstratio Math., 26(3-4), 802 - 809.

[10] Popa V (1999) Some fixed point theorems for compatible mappings satisfying an implicit relations. Demonstratio Math., 32, 157 - 163.

[11] Popa, V (2005) A generalization of Meir-Keeler type common fixed point theorem under for four non-compatible mappings. Sarajevo J. Math., 1(13), 135 - 142.

[12] Sessa S (1982) On a weak commutativity condition of mappings in fixed point considerations. Publ. Inst. Math., 32(46), 149 - 153.

[13] Sharma S and Deshpandey B (2002) On compatible mappings satisfying an implicit relation in common fixed point considerations. Tamkang J. Math., 33(3), 245 - 252. 